\title{
La motivación del docente de educación física en el aprendizaje motor de los estudiantes del nivel primario de la I.E. N²0827 "Mercedes Indacochea" de la ciudad de Huacho - 2015
}

\section{The motivation of the physical education teacher in the motor learning of the students of the I.E. N²0827 "Mercedes Indacochea" of the Huacho city - 2015 \\ Manuel Vicente Bustamante Cerna ${ }^{1}$}

\section{RESUMEN}

Objetivo: Determinar de qué manera la motivación del docente de educación física influye en el aprendizaje motor de los estudiantes del nivel primario de la I.E. № 20827 "Mercedes Indacochea” de la ciudad de Huacho. Métodos: La metodología fue de diseño no experimental, transversal, correlacional causal. Para la recolección de datos se empleó la técnica de la encuesta, utilizando como instrumento un cuestionario. La población estuvo conformada por 04 docentes de educación física y 363 estudiantes del nivel primario; asimismo la muestra fue 186 estudiantes. Resultados: Con respecto a la variable "Motivación", el 50\% manifiesta un grado motivacional aceptable y el 50\% manifiesta un grado motivacional insatisfactorio. Con respecto a la variable "Aprendizaje motor", respecto al nivel de aptitud física el $41,4 \%$ de los encuestados tienen un nivel bajo de aptitud física, el $51,6 \%$ tiene un nivel regular y el $7 \%$ un buen nivel; respecto al nivel de habilidades y destrezas el $7,5 \%$ de los encuestados tienen un nivel bajo en sus habilidades y destrezas, el $85,5 \%$ tiene un nivel regular y el $7 \%$ un buen nivel; respecto al aprendizaje motor el $5,4 \%$ de los encuestados tienen un nivel bajo en su aprendizaje motor, el $87,6 \%$ tiene un nivel regular y el $7 \%$ un buen nivel. Conclusiones: La motivación del docente de educación física influye significativamente $(p=0,000)$ en el aprendizaje motor de los estudiantes del nivel primario de la I.E. $N^{\circ} 20827$ "Mercedes Indacochea" de la ciudad de Huacho- 2015.

Palabras clave: Motivación extrínseca, motivación intrínseca, nivel de aptitud física, nivel de habilidades y destrezas, nivel perceptivo motriz.

\section{ABSTRACT}

Objective: To determine how the motivation of the physical education teacher influences the motor learning of students at the primary level of the I.E. $N^{\circ} 20827$ "Mercedes Indacochea" of the city of Huacho. Methods: The methodology was non-experimental, cross-sectional, causal correlational design. For data collection, the survey technique was used, using a questionnaire as an instrument. The population consisted of 04 physical education teachers and 363 primary level students; also the sample was 186 students. Results: With respect to the variable "Motivation", $50 \%$ show an acceptable motivational level and $50 \%$ show an unsatisfactory motivational level. Regarding the variable "Motor learning", regarding the level of physical fitness, $41.4 \%$ of those surveyed have a low level of physical fitness, $51.6 \%$ have a regular level and $7 \%$ a good level; Regarding the level of abilities and skills, $7.5 \%$ of those surveyed have a low level in their abilities and skills, $85.5 \%$ have a regular level and $7 \%$ a good level; Regarding motor learning, $5.4 \%$ of those surveyed have a low level of motor learning, $87.6 \%$ have a regular level and $7 \%$ a good level. Conclusions: The motivation of the physical education teacher influences significantly $(p=0.000)$ in the motor learning of the students of the primary level of the I.E. $N^{\circ}$ 20827 "Mercedes Indacochea" of the city of Huacho - 2015.

Keywords: Extrinsic motivation, intrinsic motivation, level of physical aptitude, level of skills and abilities, motor perceptual level.

\section{INTRODUCCIÓN}

Actualmente la Educación Física escoge los contenidos a impartir basados en los objetivos que se desean cumplir de acuerdo a las capacidades físicas eventuales y las coordinadas. La Educación Física como área curricular tiene contenidos para enseñar y eso es lo que justifica su lugar en la escuela. No se trata sólo de desarrollar aptitudes o funciones sino además hay un aprendizaje en los alumnos, una adquisición de conocimiento, por lo tanto, hay un contenido a ser atendido en su formación integral (bio-psico-social).

Con respecto a la motricidad está comprendida por el conocimiento y el desarrollo del cuerpo es decir las condiciones físicas con las que se cuenta para hacer frente a los diferentes trabajos de las distintas situaciones y las conductas lúdicas que se encargan de hacer divertidas las funciones motrices, también se considera que el aprendizaje y el desarrollo de las habilidades de movimiento ayudan a que se sea más eficiente frente a las situaciones que se viven a diario. Se considera que el niño es competente en su aprendizaje motor cuando logra estructurar su motricidad, cuando logra desarrollar su condición física, cuando emplea las técnicas de su cuerpo y sus movimientos en su entorno; esto ayuda al desarrollo lúdico, su expresión corporal, su axiología corporal, y en su manera de relacionarse con su entorno y consigo mismo. Todo ésta actividad repercute de manera positiva en su calidad de vida, en su desempeño estudiantil, en el empleo de su tiempo libre, así como en sus hábitos de vida.

Prado (2005) refiere que, la motivación es aquel procedimiento que realizar la mediación que estimula al cuerpo, que está relacionado con una acción o respuesta en particular. Es por ello que se debe tener claro que la motivación es la energía que se encarga de impulsar a que la persona desee realizar algo, ésta motivación puede tener dos direcciones la que impulsa y encamina al éxito o al fracaso lo cual se vincula a la alegría o tristeza.

Cañizares, y Carbonero, (2017) señalan que, el aprendizaje motor son secuencias de acciones musculares hechas con alto grado de competencia. Este proceso no es muscular, sino neural ya que los músculos son meros ejecutores de órdenes mandas del cerebro y es allí donde se produce el aprendizaje.

Recibido:16/10/2020 - Aprobado:15/11/2020

Universidad Nacional Mayor de San Marcos (Lima - Perú). 
Chandi y Osorio (2015) refieren que la motivación es parte importante en el proceso del aprendizaje, pero en igual medida es esencial considerar las necesidades e intereses que presente el estudiante, así como los factores personales, sociales e institucionales, pues estos elementos en su conjunto garantizan no solo un aprendizaje sino también que este sea significativo.

Vivar (2015), manifiesta que no puede influir un ambiente de aprendizaje porque no se cuenta con el mismo y, por ende, el desarrollo de la psicomotricidad es relativa.

Zevallos (2016), demuestra en los resultados de su investigación que, el aprendizaje motor revela que la motivación influye de manera significativa en las dimensiones: cognitiva, asociativa y autonomía del aprendizaje motor de los estudiantes del cuarto grado de instituciones educativas ex variante técnica del cono sur de la ciudad de Juliaca. Envuelve de forma directa y comprometida en su esquematización de imágenes motrices, la cual dirige la conducta a través de la práctica para el logro de los objetivos, generando así un nivel de logro condicionado en los aprendizajes esperados de los estudiantes.

Miranda (2016), manifiesta que, la correlación entre la motivación y estrategias de aprendizaje es de manera directa, esto quiere decir, que a mejor motivación mejor serán las estrategias de aprendizaje de los estudiantes, mientras que Sachun (2017), refiere que si existe correlación entre la motivación y los estilos de aprendizaje en los estudiantes.

Es así que en la presente investigación se estudia de qué manera la motivación del docente de educación física influye en el aprendizaje motor de los estudiantes del nivel primario de la I.E. $N^{\circ} 20827$ Mercedes Indacochea, Huacho.

\section{MATERIAL Y MÉTODOS}

La metodología corresponde a un diseño no experimental, transversal, correlacional causal. Los instrumentos utilizados fueron un cuestionario y una ficha de observación. El primer instrumento ayudó a medir la variable "Motivación"; mientras que el segundo ayudó a medir la variable "Aprendizaje motor". El procesamiento de la información se realizó mediante el análisis de la técnica estadística descriptiva de distribución de frecuencias y para identificar el grado de correlación de las variables se usó la técnica de correlación del chi cuadrado.

\section{RESULTADOS}

Análisis de la variable "Motivación"

Tabla 1 Nivel de motivación de los docentes de Educación Física de la I.E. N ${ }^{\circ} 20827$

\begin{tabular}{cccc} 
& & Frecuencia & Porcentaje \\
\hline \multirow{2}{*}{ Validos } & Aceptable & 2 & 50,0 \\
& Insatisfecho & 2 & 50,0 \\
\hline & Total & 4 & 100,0 \\
\hline
\end{tabular}

Se puede apreciar en la Tabla 1 que el $50 \%$ manifestó un grado motivacional aceptable y el otro $50 \%$ presentó un grado motivacional insatisfactorio.

Tabla 2 Nivel de motivación extrínseca de los docentes de Educación Física de la IE $N^{\circ} 20827$

\begin{tabular}{cccc} 
& & Frecuencia & Porcentaje \\
\hline \multirow{2}{*}{ validos } & Insatisfecho & 2 & 50,0 \\
& Aceptable & 1 & 25,0 \\
& Satisfecho & 1 & 25,0 \\
\hline \multirow{2}{*}{ Total } & 4 & 100,0 \\
\hline
\end{tabular}

Se observa en la Tabla 2 que el $50 \%$ de los docentes manifestaron un grado motivacional insatisfactorio, el $25 \%$ presentó un grado motivacional aceptable y el otro $25 \%$ indicaron un grado motivacional satisfactorio.

Tabla 3 Nivel de motivación intrínseca de los docentes de Educación Física de la IE N²0827

\begin{tabular}{cccc} 
& & Frecuencia & Porcentaje \\
\hline \multirow{3}{*}{ Validos } & Aceptable & 1 & 25,0 \\
& Insatisfecho & 2 & 50,0 \\
& Satisfecho & 1 & 25,0 \\
\hline & Total & 4 & 100,0 \\
\hline
\end{tabular}

Se observa en la Tabla 3 que el $50 \%$ de los encuestados indicaron un grado motivacional insatisfactorio, el $25 \%$ presentó un grado motivacional aceptable y el otro $25 \%$ indicaron un grado motivacional satisfactorio

\section{Resultado de la variable "Aprendizaje motor"}

Tabla 4 Nivel de aptitud física de los estudiantes

\begin{tabular}{cccc} 
& & Frecuencia & Porcentaje \\
\hline \multirow{3}{*}{ Validos } & Bajo & 7 & 4,4 \\
& Regular & 96 & 51,6 \\
& Bueno & 13 & 7,0 \\
\hline & Total & 186 & 100,0 \\
\hline
\end{tabular}

En la Tabla 4 se aprecia que el $41,4 \%$ de los encuestados tienen un nivel bajo de aptitud física, el $51,6 \%$ tuvieron un nivel regular y el $7 \%$ un buen nivel

Tabla 5 Nivel de habilidades y destrezas de los estudiantes

\begin{tabular}{cccc} 
& & Frecuencia & Porcentaje \\
\hline \multirow{3}{*}{ Validos } & Bajo & 14 & 7,5 \\
& Regular & 159 & 85,5 \\
& Bueno & 13 & 7,0 \\
\hline & Total & 186 & 100,0 \\
\hline
\end{tabular}

Se observa en la Tabla 5 que el $7,5 \%$ de los encuestados tuvieron un nivel bajo en sus habilidades y destrezas, el $85,5 \%$ tuvo un nivel regular y el $7 \%$ un buen nivel 
Tabla 6 Aprendizaje motor de los estudiantes

\begin{tabular}{cccc}
\hline & & Frecuencia & Porcentaje \\
\hline \multirow{3}{*}{ Validos } & Bajo & 10 & 5,4 \\
& Regular & 163 & 87,6 \\
& Bueno & 13 & 7,0 \\
\hline \multirow{3}{*}{ Total } & 186 & 100,0 \\
\hline
\end{tabular}

Se observa en la Tabla 6 que el 5,4\% de los encuestados tuvieron un nivel bajo en su aprendizaje motor, el $87,6 \%$ tuvieron un nivel regular y el $7 \%$ un buen nivel.

\section{DISCUSIÓN DE RESULTADOS}

En cuanto a la Hipótesis General, La motivación del docente de educación física influye significativamente en el aprendizaje motor de los estudiantes del nivel primario de la I.E. N ${ }^{\circ} 20827$ "Mercedes Indacochea" de la ciudad de Huacho - 2015, donde se aprecia el resultado (X2: 36,306a ; gl: 4; p: 0.00) con el cual se acepta la hipótesis alterna y se rechaza la hipótesis nula, de tal manera (Zevallos , 2016) en su investigación "Influencia en la motivación en el aprendizaje motor del estudiante del cuarto de instituciones educativas Ex Variante Técnica Cono Sur Juliaca, 2015 mencionó que los estudiantes que tienen muy alto grado de motivación presentan un nivel muy bueno de aprendizaje motor, a diferencia de que tienen bajo grado de motivación expresan niveles deficientes de aprendizaje motor. Se evidencia que, la motivación influye significativamente en el aprendizaje motor de los discentes del cuarto grado de las instituciones educativas ex variante técnica del cono sur de la ciudad de Juliaca.

\section{CONCLUSIONES}

La motivación extrínseca e intrínseca del docente de educación física influye significativamente en el aprendizaje motor de los estudiantes del nivel primario de la I.E. N ${ }^{\circ} 20827$ de la ciudad de Huacho.

Se evidenció que, si los docentes de educación física no logran que los estudiantes sean independientes y espontáneos, o que valoren prender como parte de su progreso personal el desarrollo del aprendizaje motor en los niños del nivel primario puede repercutir de manera negativa ocasionando en algunos casos en su salud enfermedades metabólicas como la obesidad, la diabetes, la hipertensión arterial y estrés debido al sedentarismo.

\section{RECOMENDACIONES}

Fomentar a que los estudiantes realicen sus ejercicios de manera activa y con placer para motivar a que sean independientes y espontáneos en su aprendizaje al cual les facilitaran la activación de sus componentes emocionales, cognitivos y motrices.

Utilizar materiales necesarios al momento de realizar los ejercicios físicos para motivar a que los alumnos sean independientes y espontáneos en su aprendizaje que le facilitaran la activación de sus componentes emocionales, cognitivos y motrices.

\section{REFERENCIAS.}

Cañizares, J., \& Carbonero, C. (2017). Cómo mejorar el aprendizaje motor de tu hijo. España: Wanceulen Editorial.

Prado, J. (2005). Psicología. Lima: Escuela Nueva S.A.

Chandi, K., \& Osorio, J. (2015). Motivación para el aprendizaje en estudiantes de la Universidad de Cuenca. Tesis de grado, Universidad de Cuenca. Recuperado el 04 de S e p t i e m b r e d e $2018, \quad d$ e http://dspace.ucuenca.edu.ec/bitstream/123456789/23 533/1/tesis.pdf

Miranda, D. (2016). Estrategias de aprendizaje y motivación en estudiantes de una institución pública de Nuevo Chimbote. Tesis grado, Universidad César Vallejo. Recuperado el 04 de Septiembre de 2018, de http://repositorio.ucv.edu.pe/bitstream/handle/UCV/406/ mirando cd.pdf?sequence $=1$ \&isAllowed $=y$

Sachun, C. (2017). Motivación y los estilos de aprendizaje en estudiantes de secundaria de las instituciones públicas del distrito de Coishco. Tesis de grado, Universidad César Vallejo. Recuperado el 04 de Septiembre de 2018, d

http://repositorio.ucv.edu.pe/bitstream/handle/UCV/413/ sachun_rc.pdf?sequence $=1$

Vivar, A. (2015). Importancia del desarrollo psicomotriz y su influencia en los diferentes ambientes de aprendizaje en los niños y niñas de 4 y 5 años del centro de desarrollo infantil Acuarela del Cantón la Troncal durante el periodo del mes de agosto hasta el mes de dici. Tesis de grado, Universidad Católica de Cuenca, Ecuador. Recuperado e I 22 d e A bril de 2018, d e http://dspace.ucacue.edu.ec/bitstream/reducacue/7352 /3/TESIS\%20AMELIA\%20VIVAR\%20CONDO.pdf

Zevallos, J. (2016). Influencia en la motivación en el aprendizaje motor del estudiante del cuarto de instituciones educativas Ex Variante Técnica Cono Sur Juliaca 2015. Tesis de grado, Universidad Andina Néstor Cáceres Velázquez, Perú. Recuperado el 22 de Abril de 2018 , d e http://repositorio.uancv.edu.pe/bitstream/handle/UANC V/766/TESIS\%20T036_02146584_M.pdf?sequence=1 \&isAllowed=y 